Instituto Internacional de Investigación y Desarrollo Tecnológico Educativo INDTEC, C.A.

DOI: https://doi.org/10.29394/Scientific.issn.2542-2987.2021.6.19.12.243-262

OAI-PMH: http://www.indteca.com/ojs/index.php/Revista Scientific/oai

Artículo Original / Original Article

\title{
Repercusión SARS-CoV-2 en salud mental y bienestar psicológico del personal Centro de Salud Huambalo 2020
}

\author{
Autores: Pablo Xavier Pérez Chipantiza \\ Universidad Regional Autónoma de Los Andes, UNIANDES \\ pg.pabloxpc00@uniandes.edu.ec \\ Ambato, Ecuador \\ https://orcid.org/0000-0002-3382-9355 \\ Darwin Raúl Noroña Salcedo \\ Universidad Regional Autónoma de Los Andes, UNIANDES \\ darwin norona@yahoo.com \\ Ambato, Ecuador \\ https://orcid.org/0000-0002-0630-0456 \\ Vladimir Vega Falcón \\ Universidad Regional Autónoma de Los Andes, UNIANDES \\ vega.vladimir@gmail.com \\ Ambato, Ecuador \\ https://orcid.org/0000-0003-0140-4018
}

\section{Resumen}

Este trabajo de investigación tiene como antecedente que, en diciembre del año 2019 en Wuhan, Ciudad en China, aparece un nuevo virus, que en febrero del año 2020 llega al Ecuador, el Centro de Salud Huambaló, presentó los primeros casos, comprometiendo al personal de atención directa. La pandemia, incidió en la salud mental y bienestar psicológico de los profesionales, en los indicadores positivos de salud como creatividad, autoestima, consciencia social y proyectos de vida, fueron afectados. El objetivo del estudio fue analizar la repercusión SARS-CoV-2 en la salud mental y bienestar psicológico del personal Centro de Salud Huambaló en el año 2020. Fue un estudio cuantitativo, no experimental, transversal, descriptivo, que se practicó a cincuenta (50) trabajadores del Centro de Salud Huambaló de Ecuador. Con el Cuestionario Salud General Goldberg para identificar problemas psicosociales, somáticos, psicológicos y conductuales; análisis descriptivo e inferencial se realiza en SPSS, prueba Chi cuadrada Pearson para analizar la relación entre variables. La exposición autopercibida al SARS-CoV-2 produjo mayor tensión emocional, con detrimento de la evaluación en salud mental y bienestar psicológico por debajo de la media esperada en dimensiones de disfunción social y cargo. El estudio evidenció que la repercusión SARS-CoV-2 ha ocasionado efectos de salud en dimensiones somáticas, ansiedad, depresión y función social; comprobando deterioro en la satisfacción de actividades diarias, confianza, miedo ante el desempeño de las responsabilidades, sensación de enfermedad, cefaleas, y cansancio generalizado.

Palabras clave: repercusión; salud mental; bienestar; psicológico; sars-cov-2.

\section{Cómo citar este artículo:}

Pérez, P., Noroña, D., \& Vega, V. (2021). Repercusión SARS-CoV-2 en salud mental y bienestar psicológico del personal Centro de Salud Huambalo 2020. Revista Scientific, 6(19), 243-262, eISSN: 2542-2987. Recuperado de: https://doi.org/10.29394/Scientific.issn.25422987.2021.6.19.12.243-262

Fecha de Recepción: 18-09-2020
Fecha de Aceptación: 16-12-2020
Fecha de Publicación: 05-02-2021 
OAl-PMH: http://www.indteca.com/ojs/index.php/Revista Scientific/oai

Artículo Original / Original Article

\title{
SARS-CoV-2 impact on mental health and psychological well-being of the staff Huambalo Health Center 2020
}

\begin{abstract}
This research work has as a precedent that, in December 2019 in Wuhan, a city in China, a new virus appears, which in February 2020 reaches Ecuador, the Huambaló Health Center, presented the first cases, compromising the staff direct care. The pandemic affected the mental health and psychological well-being of professionals, positive health indicators such as creativity, self-esteem, social awareness and life projects were affected. The objective of the study was to analyze the SARS-CoV-2 impact on the mental health and psychological well-being of the Huambaló Health Center staff in 2020. It was a quantitative, non-experimental, cross-sectional, descriptive study, which was carried out on fifty (50) workers at the Huambaló Health Center in Ecuador. With the Goldberg General Health Questionnaire to identify psychosocial, somatic, psychological and behavioral problems; Descriptive and inferential analysis is performed in SPSS, Pearson's Chi square test to analyze the relationship between variables. Self-perceived exposure to SARSCoV-2 produced greater emotional stress, to the detriment of the evaluation in mental health and psychological well-being below the expected mean in dimensions of social dysfunction and position. The study showed that the SARS-CoV-2 repercussion has caused health effects in somatic dimensions, anxiety, depression and social function; verifying deterioration in the satisfaction of daily activities, confidence, fear of the performance of responsibilities, feeling of illness, headaches, and generalized fatigue.
\end{abstract}

cov-2.

Keywords: repercussion; mental health; wellness; psychological; sars-

\footnotetext{
How to cite this article:

Pérez, P., Noroña, D., \& Vega, V. (2021). SARS-CoV-2 impact on mental health and psychological well-being of the staff Huambalo Health Center 2020. Revista Scientific, 6(19), 243-262, e-ISSN: 2542-2987. Recovered from: https://doi.org/10.29394/Scientific.issn.2542-2987.2021.6.19.12.243$\underline{262}$
}

Date Received: 18-09-2020
Date Acceptance: 16-12-2020
Date Publication: 05-02-2021 


\section{Introducción}

La temática de salud mental en los últimos años ha tenido cierta relevancia en diferentes aspectos de la comunidad, y deja de ser simplemente una ciencia aislada para posesionarse en los diferentes espacios que la sociedad le permite, dejando su invisibilidad y rompiendo los estigmas que la misma ha generado entre las personas.

Paradójicamente, el sitio donde menos importancia se le ha concedido a la salud mental y el bienestar psicológico, es justamente al personal que labora en esta área, donde por su contexto se ven abismalmente afectados, según Ramírez-Ortiz, Castro-Quintero, Lerma-Córdoba, Yela-Ceballos y Escobar-Córdoba (2020): el hecho de enfrentarse a una enfermedad caracterizada por su transmisibilidad y letalidad, generando temor, angustia e impotencia de no poder acogerse a las medidas de aislamiento social como media preventiva debido al ser parte de los profesionales de primera línea de atención.

El desconocimiento de protocolos de actuación claros, así como medidas de prevención y protección para evitar el contagio, sumado a la falta de cultura en la observancia de normas de las personas para evitar la propagación del virus. Las repercusiones que esta pandemia pudo haber causado más allá de los padecimientos físicos relacionados con las medidas restrictivas de cuarentena, distanciamiento social, aislamiento, interrupción de los normales servicios en la atención de salud, es el hecho de analizar repercusión de SARS-CoV-2 en el personal de primera línea de atención.

De acuerdo con Acosta-Quiroz y Iglesias-Osores (2020a): conforme fueron extendiéndose los casos por el territorio el equipo de salud conformados por médicos, enfermeros, laboratoristas, imagenólogos y todo trabajador relacionado con el área de salud en general se vieron expuestos a un riesgo incrementado, lo que generó estados de ansiedad, incertidumbre, estrés por la idea persistente de contagio. 
Señala Acosta-Quiroz y Iglesias-Osores (2020b): que las consecuencias que podría conllevar a corto y mediano plazo sobre el personal sanitario con sintomatología depresiva, ansiosa, alteraciones del sueño, ataques de pánico se relacionarán a su salud mental como parte un estrés postraumático, por eso el hecho de cuidar del bienestar psicológico de equipo de salud tiene una gran importancia, debiéndose monitorear al recurso humano para evitar complicaciones en su psiquis.

Los profesionales de la salud por su formación y la línea de ciencia que eligieron se podría asumir que los mismo están preparados para enfrentar situaciones que conlleven perdida, ansiedad, miedo, tristeza, preocupación que dentro de su área laboral podría considerarse como algo normal, pero cuando se agrega el componente de lo desconocido y que este pueda afectar no solo así mismo sino a su entorno más cercano, la percepción cognitiva cambia, su bloqueo de autoinmunidad empieza a desaparecer y vuelve a la persona emocionalmente más susceptible.

Para la Organización Panamericana de la Salud (OPS/OMS, 2016): al convertirse en una amenaza la pandemia del SARS-CoV-2, no solo para la salud física sino para la salud mental de las personas en especial de aquellos que se encuentran en primera línea, se convierte en una prioridad de atención por el impacto que este puede ocasionar en el ámbito psicosocial del individuo, siendo recomendable las intervenciones en salud mental y apoyo psicológico individual y colectivo.

En concordancia con la Organización Panamericana de la Salud (OPS, 2020a): se establecieron medidas de prevención como la cuarentena, distanciamiento social, aislamiento, con elementos que incrementaron la letalidad del problema como la falta de medicamentos, equipos de protección, áreas de atención hospitalaria, que a la par aumentó la sinergia del contagio individual y colectivo, lo que desencadena miedo, tristeza, ansiedad ahondando las alteraciones psicosociales. 
A pesar de las advertencias de los organismos internacionales de mantenerse preparados ante posibles pandemias de riesgo biológicos y evitar estar desprevenidos, de acuerdo con la Organización Panamericana de la Salud (OPS, 2020b): manteniendo un sistema educomunicacional con la población que le permita conocer la magnitud y consecuencia de una enfermedad, así como las medidas preventivas, en los referente a salud física y mental, finalmente termina relegado en la planificación de actuación aspectos concernientes a la salud mental y bienestar psicológico de las personas.

Los profesionales dentro del ámbito de salud se convirtieron en uno de los recursos humanos que por su formación debían permanecer en exposición frente al virus. Ante esta situación, para Álvarez y Donisio (2020): el personal de salud incrementa su vulnerabilidad y consecuentemente se hace necesario identificar las reacciones psicológicas de los mismos y determinar el impacto para establecer mecanismos de actuación.

Autores como García-Iglesias, Gómez-Salgado, Martín-Pereira, Fagundo-Rivera, Ayuso-Murillo, Martínez-Riera y Ruiz-Frutos (2020): la pandemia ha generado diversos cambios a todo nivel desde lo económico que resulta ser el más notable, pero también en el ámbito social y cultural con el uso obligatorio de mascarillas, así como el distanciamiento social, medidas de higiene como la desinfección de manos y espacios de uso común. Finalmente, el sector salud ha incrementado un sistema educomunicacional para fortalecer las medidas de bioseguridad en la atención que se brinda a los pacientes.

En hechos anteriores similares, manifiesta Huarcaya-Victoria (2020a): los equipos de primera intervención han sido los primeros en desarrollar inquietudes, ansiedad, miedo, temor a fallecer desplegando impresiones de soledad, desconsuelo e irritabilidad, a pesar de la experiencia vivida. Las investigaciones al respecto se han enfocado al aspecto clínico y epidemiológico, así como las políticas públicas para enfrentarlos, sin que se 
haya otorgado relevancia a la salud mental de este grupo de trabajo.

Al respecto, existen algunos instrumentos de validación psicológica que permite evaluar, diagnosticar, orientar y guiar una investigación respecto a la temática propuesta, bajo el análisis de las principales causalidades que pudo haberse presentado como repercusión a la pandemia SARS-CoV-2. De acuerdo con el estudio de Parrado-González y León-Jariego (2020): el efecto medido a través de la Escala de Impacto de Evento-Revisada (IES-R), en la cual se obtuvo un valor de 0,896 al aplicarse el alfa de Cronbach dentro del análisis de la consistencia, que midió la homogeneidad de lo enunciado de los instrumentos, la fiabilidad de los mismos un valor de 0,812 para la intrusión; 0,788 para la evitación y 0,769 para la hiperactivación, mientras que el estado de salud mental tuvo un valor de alfa de Cronbach de 0,869 mediante el Cuestionario de Salud General de Goldberg (GHQ-12).

Por otro lado, Becerra y Ybaseta (2020): evaluó de forma aleatoria las variables respecto a la autopercepción de su salud mediante el estadístico Chi cuadrado, los resultados son significativos con $p<0,05$, para su análisis utilizo el programa SPSS.

La importancia de realizar esta investigación es describir la repercusión de la pandemia SARS-CoV-2 en la salud mental y el bienestar psicológico del personal del centro de salud tipo B Huambaló en el año 2020, que estuvo en primera línea de atención y cuyos primeros casos fueron evidentes en la parroquia Huambaló, Ecuador, siendo consecuentemente identificados y monitoreados por el personal, lo que desarrolla un nivel investigativo de tipo descriptivo encaminada en la línea de investigación en factores de riesgo psicosocial y su impacto en el clima laboral.

El objetivo del estudio fue analizar el impacto del virus SARS-CoV-2 del personal profesional en el Centro de Salud Huambaló de Ecuador, dentro del punto de vista de salud mental y psicológico en el año 2020. 


\section{Metodología}

Fue un estudio con enfoque cuantitativo, diseño no experimental, descriptivo, transversal, con el propósito de analizar la repercusión de la pandemia SARS-CoV-2 en la salud mental y el bienestar psicológico se trabajó con una muestra constituida por todo el personal que labora en el Centro de Salud Tipo B Huambaló en el año 2020, conformado por 50 trabajadores. Concordando con Romero, Castro, Álvarez, Velázquez, Comas y Falcon (2017): la investigación de mantener el enfoque en la realidad regional, enfrentando un problema a través de la aplicación del método científico.

En la presente investigación no fue necesario realizar el cálculo muestral debido a que se trabajó con la totalidad de la población en estudio, la distribución que se muestra en la tabla 1; a quienes se les aplicó un cuestionario de manera digital y online cuya duración fue de aproximadamente diez minutos.

Se utilizaron criterios de inclusión dentro de los cuales están todos los trabajadores del Centro de Salud Huambalo con relación de dependencia, que consta de todos los puestos sean operativos o administrativos, que se encuentre de turno; se excluyó todo personal que se encontraban en periodo de vacaciones, de baja médica, en proceso de desvinculación con la institución, se eliminó todo personal que no haya completado de manera total la encuesta $\mathrm{GHQ}$.

Se analizaron las repercusiones debido a cuatro áreas psiquiátricas como son la depresión, la ansiedad, la inadecuación social y síntomas somáticos, aplicando un instrumento validado denominado cuestionario de Salud General de Goldberg, con la versión que consta de (28) ítems, asegurando su validez lógica y de contenido.

Para la tabulación del instrumento se empleó la hoja de cálculo Excel, y posteriormente los datos fueron llevados al programa estadístico informático SPSS para el cálculo del Chi cuadrado de Pearson ( $X^{2}$ de Pearson) y de Odds 
ratio como medida de asociación.

En referencia al tema, Galindo, Meneses, Herrera, Cabrera, Suchil, Rivera y Aguilar (2017): exponen que las propiedades psicométricas del Cuestionario de Salud General de Goldberg, ha permitido la validación en diversas poblaciones, mostrando un alfa de Cronbach entre 0,89 y $0,75(22-29)$ utilizados en varios estudios epidemiológicos para analizar el estado de salud mental en las personas.

Se utilizó la escala de Likert de cuatro respuestas (0) Bastante más que lo habitual, (0) Mucho más que lo habitual, (1) No más que lo habitual, (1) No, en absoluto, (0) Igual que lo habitual, (0) Más satisfecho que lo habitual, (1) Menos satisfecho que lo habitual, (1) Mucho menos satisfecho que lo habitual.

Una vez que desarrollaron los cuestionarios la totalidad de los trabajadores del Establecimiento de Salud, se tabularon las respuestas de acuerdo con el baremo de GHQ 28, que permite evaluar el estado de salud general de cuatro áreas psicológicas.

Con base a la evaluación del estado de salud general de cuatro áreas psicológicas se realiza la inferencia con las variables nominales con el objetivo de determinar la significancia estadística. Para tal propósito, de acuerdo con Sánchez, Vega, Gómez y Vilema (2020): se calculó el Chi Cuadrado en el que se admiten los valores por debajo a 0,05 como significantes. Una vez obtenido las inferencias se procedió a calcular mediante el mismo programa la razón de productos cruzados $(\mathrm{OR})$ que permita corroborar la asociación de las variables determinado la probabilidad de ocurrencia.

\section{Resultados}

En los datos de la tabla 1, se resalta que el mayor porcentaje de la población corresponde al sexo femenino con un $80 \%$ que se relaciona a las profesiones de enfermeros y médicos que sumados alcanza el $50 \%$, las que están enfocadas en actividades operativas con el $96 \%$, los mismos que se 
encuentran dentro de un grupo adulto joven menor de 40 años con un $88 \%$ (62\% para el grupo etario $20-30$ años y $26 \%$ del rango $31-40$ años), en concordancia con la escolaridad de tercer nivel del $70 \%$ y la experiencia que poseen en menos de 5 años con el $72 \%$ ( $38 \%$ de 1 a 5 años y $34 \%$ en menores de 1 año), lo que representa un colectivo que puede enfrentar de mejor manera las repercusiones de eventos adversos que se presentan de manera inesperada.

Tabla 1. Características sociodemográficas de los participantes.

\begin{tabular}{|c|c|c|}
\hline \multicolumn{3}{|c|}{ Variables Nominales } \\
\hline & No & $\%$ \\
\hline \multicolumn{3}{|l|}{ Sexo } \\
\hline Hombre & 10 & $20 \%$ \\
\hline Mujer & 40 & $80 \%$ \\
\hline \multicolumn{3}{|l|}{ Edad } \\
\hline $20-30$ & 31 & $62 \%$ \\
\hline $31-40$ & 13 & $26 \%$ \\
\hline $41-50$ & 4 & $8 \%$ \\
\hline más de 50 & 2 & $4 \%$ \\
\hline \multicolumn{3}{|l|}{ Estado civil } \\
\hline Casado & 20 & $40 \%$ \\
\hline Divorciado & 3 & $6 \%$ \\
\hline Soltero & 24 & $48 \%$ \\
\hline Unión Libre & 3 & $6 \%$ \\
\hline \multicolumn{3}{|l|}{ Escolaridad } \\
\hline Bachiller & 2 & $4 \%$ \\
\hline Técnico/Tecnólogo & 9 & $18 \%$ \\
\hline Tercer Nivel & 35 & $70 \%$ \\
\hline Cuarto Nivel & 4 & $8 \%$ \\
\hline \multicolumn{3}{|l|}{ Tiempo de servicio } \\
\hline más de 5 años & 14 & $28 \%$ \\
\hline de 1 a 5 años & 19 & $38 \%$ \\
\hline menor a 1 año & 17 & $34 \%$ \\
\hline \multicolumn{3}{|l|}{ Cargo } \\
\hline Administrativo & 2 & $4 \%$ \\
\hline Operativo & 48 & $96 \%$ \\
\hline \multicolumn{3}{|l|}{ Puesto } \\
\hline Analista & 1 & $2 \%$ \\
\hline Auxiliar & 2 & $4 \%$ \\
\hline Director/a & 1 & $2 \%$ \\
\hline Enfermero/a & 11 & $22 \%$ \\
\hline Médico/a & 14 & $28 \%$ \\
\hline Obstetra & 3 & $6 \%$ \\
\hline Odontólogo/a & 1 & $2 \%$ \\
\hline Psicólogo/a & 1 & $2 \%$ \\
\hline
\end{tabular}


Artículo Original / Original Article

Fuente: Los Autores (2020).

\begin{tabular}{|l|l|l|}
\hline \multicolumn{3}{|c|}{ Variables Nominales } \\
\hline Químico farmacéutico & 1 & $2 \%$ \\
\hline TAPS & 9 & $18 \%$ \\
\hline Tecnólogo Medico & 2 & $4 \%$ \\
\hline Terapista & 4 & $8 \%$ \\
\hline
\end{tabular}

Este dato guarda relación con lo que manifiesta Muñoz, Molina, Ochoa, Sánchez y Esquivel (2020): donde se evidencia que no todas las personas reaccionan igual y se deben considerar variables sociodemográficas, resaltándose el porcentaje de mujeres es mayoritariamente más elevado en varios niveles, conllevando a una sobrecarga emocional. Sin embargo, la resiliencia que suelen tener actúa como un factor protector que les permite enfrentar eventos adversos.

No obstante, en el estudio de Alomo, Gagliardi, Peloche, Somers, Alzina y Prokopez (2020): se recalca que los efectos por el SARS-CoV-2 fue mayor en mujeres con relación a los hombres, considerando que las mujeres en edad reproductiva están predispuestas a sufrir trastornos de ansiedad.

De los resultados obtenidos en la tabla 2 , se puede evidenciar que al momento no se ha encontrado asociación significativa, salvo dentro de ansiedad e insomnio con un $48 \%$ (14\% alto y $34 \%$ medio) que se relaciona con el proceso cognitivo que tiene las personas antes eventos que son de desconocimiento y el cuerpo actúa de esta manera como mecanismos de defensa, por otra parte, la depresión grave que pudiera haberse esperado solo se evidencia un $10 \%$ de aceptación ( $4 \%$ alto y $6 \%$ medio).

Tabla 2. Salud mental y el bienestar psicológico del personal.

Fuente: Los Autores (2020).

\begin{tabular}{|l|c|c|c|}
\hline \multicolumn{1}{c|}{} & Alto & Medio & Bajo \\
\hline Síntomas Somáticas & $12,0 \%$ & $30,0 \%$ & $58,0 \%$ \\
\hline Ansiedad e Insomnio & $14,0 \%$ & $34,0 \%$ & $52,0 \%$ \\
\hline Disfunción Social & $8,0 \%$ & $24,0 \%$ & $68,0 \%$ \\
\hline Depresión Grave & $4,0 \%$ & $6,0 \%$ & $90,0 \%$ \\
\hline
\end{tabular}


Dentro de los resultados que se muestran en la tabla 3 , se obtienen inferencias respecto a cargo -disfunción social con un 0,037 y cargo- depresión grave con 0,028, como se observa en lo referente a puesto-disfunción social, puesto-depresión grave, puesto-significación, formación académica-ansiedad e insomnio, formación académica-depresión grave, formación académicasignificación con los casos no se evidencia una consecuencia notoria.

Tabla 3. Inferencia estadística entre las características sociodemográficas de los participantes con la salud mental y el bienestar psicológico del personal. $\left({ }^{*}\right)$ Prueba de Chi Cuadrado, valores inferenciales por debajo de 0,05.

\begin{tabular}{|l|c|c|c|c|c|}
\hline \multicolumn{1}{l|}{} & $\begin{array}{c}\text { Síntomas } \\
\text { Somáticas }\end{array}$ & $\begin{array}{c}\text { Ansiedad e } \\
\text { Insomnio }\end{array}$ & $\begin{array}{c}\text { Disfunción } \\
\text { Social }\end{array}$ & $\begin{array}{c}\text { Depresión } \\
\text { Grave }\end{array}$ & Significación \\
\hline Edad & 0,507 & 0,937 & 0,763 & 0,849 & 0,774 \\
\hline Sexo & 0,669 & 0,260 & 0,219 & 0,094 & 0,103 \\
\hline Tiempo de servicio & 0,945 & 0,745 & 0,289 & 0,060 & 0,144 \\
\hline Cargo & 0,206 & 0,205 & $\mathbf{0 , 0 3 7}^{*}$ & $\mathbf{0 , 0 2 8}^{*}$ & 0,134 \\
\hline Puesto & 0,232 & 0,072 & $\mathbf{0 , 0 0 3}^{*}$ & $\mathbf{0 , 0 0 3}^{*}$ & $\mathbf{0 , 0 1 3}^{*}$ \\
\hline Estado civil & 0,384 & 0,244 & 0,324 & 0,986 & 0,537 \\
\hline Formación académica & 0,284 & $\mathbf{0 , 0 1 1 ^ { * }}$ & 0,359 & $\mathbf{0 , 0 1 5}^{*}$ & $\mathbf{0 , 0 0 2}^{*}$ \\
\hline
\end{tabular}

Fuente: Los Autores (2020).

Las posibilidades respecto a las inferencias encontradas que cumplen los criterios de exposición fueron cargo y disfunción social donde de acuerdo con la tabla 4, las personas que realizan actividades administrativas tienen 3,4 (OR) veces más probabilidades de padecer una repercusión en su salud referente a la disfunción social en relación con el personal operativo.

Tabla 4. Inferencia y asociación entre cargo y disfunción social.

\begin{tabular}{|c|c|c|c|}
\hline $\begin{array}{c}\text { Inferencia } \\
\text { Estadística } \\
\boldsymbol{p}\end{array}$ & \multirow{2}{*}{ Valor OR } & \multicolumn{2}{|c|}{ Intervalo de confianza } \\
\cline { 3 - 4 } & & Inferior & Superior \\
\hline 0,037 & 3,4 & 2,2 & 5,3 \\
\hline
\end{tabular}

Fuente: Los Autores (2020).

Las autoridades que suelen ponerse al frente de los procesos de 
emergencias y desastres preparan al personal sanitario para enfrentar los eventos desde el ámbito clínico-fisiológico dejando en un segundo plano y casi siempre imperceptible las repercusiones en la salud mental y el bienestar psicológico que este tipo de sucesos suele causar en las personas profesionales y no profesionales que enfrentan de manera directa o indirectamente a corto, mediano o largo plazo.

De los resultados obtenidos producto de la aplicación del cuestionario GHQ 28, donde se evaluó el estado de salud general bajo cuatro áreas psicológicas, se puede patentizar que al momento de la investigación no se ha encontrado asociación significativa, salvo en lo referente a la ansiedad e insomnio que alcanza un $48 \%$ (14\% alto y $34 \%$ medio) que se atañe con el proceso cognitivo de las personas ante eventos que son indocumentados y se activa su mecanismo de defensa.

Para Huarcaya-Victoria (2020b): la confianza resulta un aspecto determinante para mantener el bienestar psicológico mejorando notablemente su motivación que se refleja en la calidad de atención durante una crisis sanitaria. De igual manera Martínez-Taboas (2020): señala que el aislamiento, el distanciamiento social y la cuarentena son factores que propician la aparición de sintomatología como ansiedad y depresión, resaltando la importancia de la conexión social que permite mitigar las consecuencias en la salud mental y el bienestar psicológico de las personas.

Respecto a la evaluación del estado de salud general de cuatro áreas psicológicas donde se realiza las inferencias con las variables nominales con el objetivo de determinar la significancia estadística, los resultados muestran inferencias respecto a cargo disfunción social y depresión grave que se encuentran relacionados con el hecho de que el $96 \%$ es personal operativo y solo un $4 \%$ es administrativo, respecto a las demás inferencias encontradas donde no se evidencia consecuencia notoria. 
En la aportación de Martínez-Pérez, Rivas-Laguna, Bermudez-Cordoví, Gutiérrez-Favier y Rivero-Rodríguez (2020): se encuentra una alta relación entre la enfermedad mental y sintomatología psicológica como la depresión, ansiedad y estrés. Coincidiendo con Galindo-Vázquez, Ramírez-Orozco, Costas-Muñiz, Mendoza-Contreras, Calderillo-Ruíz y Meneses-García (2020): los profesionales de atención en primera línea y el personal administrativo son más vulnerables a sobrellevar padecimientos de origen mental.

Durante el análisis estadístico de las inferencias, estas cumplen los criterios de exposición en lo referente al cargo y disfunción social, donde las personas que realizan actividades administrativas tienen 3,4 veces más probabilidades de padecer una repercusión en su salud referente a la disfunción social, en comparación con el personal operativo.

De acuerdo con la investigación de Lozano-Vargas (2020), los niveles de estrés y ansiedad poseen una elevada incidencia sobre todo en el personal de enfermería. Coincidiendo con Santamaría, Ozamiz-Etxebarria, Redondo, Alboniga-Mayor y Picaza (2020), los datos revelan que el personal sanitario tiende a padecer sintomatología depresiva, ansiosa y alteración en el patrón del sueño.

\section{Conclusiones}

La repercusión de la pandemia SARS-CoV-2 de acuerdo con la autopercepción de los trabajadores respecto a los síntomas presentes que ha ocasionado efectos bajos en la salud referente a las dimensiones somáticas, trastornos del sueño, en la ansiedad, depresión y en la función social de los trabajadores investigados. La ansiedad e insomnio mantuvieron una inferencia con respecto a la profesión de médicos y enfermeras, preponderando en el género femenino. La confluencia del bienestar psicológico y las características sociodemográficas no evidenció asociaciones significativas en los trabajadores del Centro de Salud Huambalo. 
Se ha comprobado un deterioro en la dimensión de disfunción social que se relaciona con la percepción de satisfacción de las actividades diarias, en la confianza y fe en sí mismo, mayor miedo ante el desempeño de las responsabilidades, sensación de enfermedad, dolores de cabeza, y cansancio generalizado.

EI SARS-CoV-2 ha repercutido de forma no significativa en la salud mental a corto plazo, sobre todo en el personal médico y de enfermería que resultaron los más expuestos, la muestra poblacional debería incrementarse para explicar de mejor manera el impacto de la pandemia a largo plazo.

Los resultados podrían compararse con establecimientos de salud del mismo nivel cuyas características laborales sean similares, permitiendo generar un plan de acción en salud mental con técnicas de afrontamiento para mitigar respuestas emocionales patológicas.

Las inferencias de cargo y disfunción social establecieron que el grupo que tiene más probabilidad de padecer consecuencias es el personal administrativo, por lo que se debería generar espacios para manejo de grupos terapéuticos con relación a las emociones vividas mediante la técnica de defusing y debriefing, proveyéndoles estrategias de manejo en repercusiones psicológicas.

\section{Referencias}

Acosta-Quiroz, J., \& Iglesias-Osores, S. (2020a,b). Salud mental en trabajadores expuestos a COVID-19. Revista de Neuro-Psiquiatría, 83(3), ISSN: 0034-8597. Recuperado de:

http://www.scielo.org.pe/scielo.php?script=sci arttext\&pid=S0034$\underline{85972020000300212}$

Alomo, M., Gagliardi, G., Peloche, S., Somers, E., Alzina, P., \& Prokopez, C. (2020). Efectos psicológicos de la pandemia COVID-19 en la población general de Argentina. Revista de la Facultad de Ciencias 
Médicas de Córdoba, 77(3), 176-181, e-ISSN: 1853-0605. Recuperado de: https://doi.org/10.31053/1853.0605.v77.n3.28561

Álvarez, G., \& Donisio, N. (2020). Reacciones psico-conductuales de los profesionales de salud ante una catástrofe: Una aproximación a los efectos del SARS cov2 en los equipos de salud de Córdoba. Revista de Salud Pública, 24(2), 75-77, e-ISSN: 1852-9429. Recuperado de: https://doi.org/10.31052/1853.1180.v24.n2.29195

Becerra, B., \& Ybaseta, J. (2020). Salud Autopercibida en Trabajadores de Enfermería del Primer Nivel de Atención, Durante la Pandemia de COVID-19. Revista Médica Panacea, 9(2), 113-117, e-ISSN: 22256989. Recuperado de: https://doi.org/10.35563/rmp.v9i2.330

Galindo, O., Meneses, A., Herrera, Á., Cabrera, P., Suchil, L., Rivera, L., \& Aguilar, J. (2017). Propiedades psicométricas del Cuestionario General de Salud de Goldberg -GHQ-28- en cuidadores primarios informales de pacientes con cáncer. Psicooncología, 14(19), 71-81, e-ISSN: 1696-7240. Recuperado de:

https://doi.org/10.5209/PSIC.55812

Galindo-Vázquez, O., Ramírez-Orozco, M., Costas-Muñiz, R., MendozaContreras, L., Calderillo-Ruíz, G., \& Meneses-García, A. (2020). Síntomas de ansiedad, depresión y conductas de autocuidado durante la pandemia de COVID-19 en la población general. Gaceta Médica México, 156, 298-305, e-ISSN: 2696-1288. Recuperado de: https://doi.org/10.24875/GMM.20000266

García-Iglesias, J., Gómez-Salgado, J., Martín-Pereira, J., Fagundo-Rivera, J., Ayuso-Murillo, D., Martínez-Riera, J., \& Ruiz-Frutos, C. (2020). Impacto del SARS-CoV-2 (Covid-19) en la salud mental de los profesionales sanitarios: una revisión sistemática. Revista Española de Salud Pública, 94, 1-20, e-ISSN: 2173-9110. Recuperado de: 
Huarcaya-Victoria, V. (2020a,b). Consideraciones sobre la salud mental en la pandemia de COVID-19. Revista Peruana de Medicina Experimental y Salud Publica, 37(2), 327-334, e-ISSN: 1726-4642. Recuperado de: https://doi.org/10.17843/rpmesp.2020.372.5419

Lozano-Vargas, A. (2020). Impacto de la epidemia del Coronavirus (COVID19) en la salud mental del personal de salud y en la población general de China. Revista de Neuro-Psiquiatria, 83(1), 51-56, e-ISSN: 1609-7394. Recuperado de: https://doi.org/10.20453/rnp.v83i1.3687 Martínez-Taboas, A. (2020). Pandemias, COVID-19 y Salud Mental: ¿Qué Sabemos Actualmente?. Revista Caribeña de Psicología, 4(2), 143152, e-ISSN: 2689-8535. Recuperado de:

https://doi.org/10.37226/rcp.v4i2.4907

Martínez-Pérez, J., Rivas-Laguna, Y., Bermudez-Cordoví, L., Gutiérrez-Favier, E., \& Rivero-Rodríguez, I. (2020). Efectos de la COVID-19 sobre estados afectivos emocionales de la población adulta de Puerto Padre. Revista Electrónica Dr. Zoilo E. Marinello Vidaurreta, 45(6), 1-9, e-ISSN: 1029-3027. Recuperado de:

http://revzoilomarinello.sld.cu/index.php/zmv/article/view/2388

Muñoz, S., Molina, D., Ochoa, R., Sánchez, O., \& Esquivel, J. (2020). Estrés, respuestas emocionales, factores de riesgo, psicopatología y manejo del personal de salud durante la pandemia por COVID-19. Acta Pediátrica de México, 41(4), (S127-S136), e-ISSN: 2395-8235. Recuperado de: http://dx.doi.org/10.18233/APM41No4S1ppS127$\underline{\mathrm{S} 1362104}$

OPS/OMS (2016). Protección de la Salud Mental y Atención Psicosocial en Situaciones de Epidemias. THS/MH/06/1. Washington D.C., Estados Unidos: Unidad de Salud Mental y Uso de Sustancias; Organización Panamericana de la Salud.

OPS (2020a,b). COVID-19: Intervenciones Recomendadas en Salud 
Artículo Original / Original Article

Mental y Apoyo Psicosocial (SMAPS) durante la Pandemia. OPS/NMH/NM/COVID-19/20-0026. Washington D.C., Estados Unidos: Organización Panamericana de la Salud.

Parrado-González, A., \& León-Jariego, J. (2020). COVID-19: Factores asociados al malestar emocional y morbilidad psíquica en población española. Revista Española de Salud Pública, 94, 1-16, eISSN: 2173-9110. Recuperado de: http://hdl.handle.net/10272/18350 Ramírez-Ortiz, J., Castro-Quintero, D., Lerma-Córdoba, C., Yela-Ceballos, F., \& Escobar-Córdoba, F. (2020). Consecuencias de la pandemia de la COVID-19 en la salud mental asociadas al aislamiento social. Revista Colombiana de Anestesiología, 48(4), e-ISN: 2422-0248. Recuperado de: https://doi.org/10.5554/22562087.e930

Romero, A., Castro, F., Álvarez, G., Velázquez, M., Comas, R., \& Falcon, V. (2017). La investigación científica y las formas de titulación, aspectos conceptuales y prácticos. ISBN: 978-9942-974-36-5. Ecuador: Universidad Regional Autónoma de los Andes.

Santamaría, M., Ozamiz-Etxebarria, N., Redondo, I., Alboniga-Mayor, J., \& Picaza, M. (2020). Impacto psicológico de la COVID-19 en una muestra de profesionales sanitarios españoles. Revista de Psiquiatría y Salud Mental, 1-7, e-ISSN: 1888-9891. Recuperado de: https://doi.org/10.1016/j.rpsm.2020.05.004

Sánchez, B., Vega, V., Gómez, N., \& Vilema, G. (2020). Estudio de casos y controles sobre factores de riesgo de diabetes mellitus tipo 2 en adultos mayores. Revista Universidad y Sociedad, 12(4), 156-164, eISSN: 2218-3620. Recuperado de:

https://rus.ucf.edu.cu/index.php/rus/article/view/1626 


\section{Artículo Original / Original Article}

\section{Pablo Xavier Pérez Chipantiza e-mail: pg.pabloxpc00@uniandes.edu.ec}

Nacido en Ambato, Ecuador, el 30 de junio del año 1978.

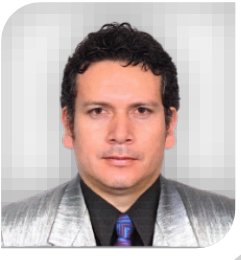

Psicólogo General por la Universidad Tecnológica Indoamérica (UTI); Licenciado en Enfermería por la Universidad Técnica de Ambato (UTA); Diplomado en Administración de Riesgos Laborales por la Universidad Técnica de Cotopaxi (UTC); Diplomado Internacional en Criminalística y Forense de la Wens Capacitaciones; Egresado de la Maestría de Salud Ocupacional de la Universidad Regional Autónoma de Los Andes (UNIANDES); Especialización en Psicología de Emergencias y Desastres de la Universidad Católica de Cuenca (UCACUE); Especialista de Promoción e Igualdad de la Salud del Ministerio de Salud Pública del Ecuador; Docente de Universidad Regional Autónoma de Los Andes (UNIANDES); Docente de la Universidad Técnica de Ambato (UTA), Ecuador. 


\section{Darwin Raúl Noroña Salcedo}

e-mail: darwin norona@yahoo.com

Nacido en Quito, Ecuador, el 13 de marzo del año 1983.

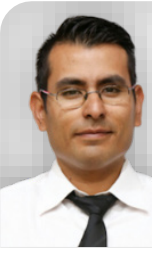

Doctor PhD. en Ciencias de la Salud Ocupacional por la Universidad de Guadalajara (UDG), México; Magister en Seguridad y Salud Ocupacional por la Universidad Central del Ecuador (UCE); Psicólogo Industrial por la UCE; Licenciado en Ciencias de la Educación mención idioma inglés por la UCE; Consultor Seguridad y Salud Ocupacional; Capacitador en Clima y Cultura Laboral; Analista de Talento Humano; Docente de la Universidad Regional Autónoma de Los Andes (UNIANDES), Ecuador. 


\section{Vladimir Vega Falcón \\ e-mail: vega.vladimir@gmail.com}

Nacido en Matanzas, Cuba, el 18 de agosto del año

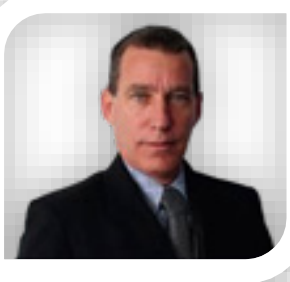

1964. Licenciado en Contabilidad por la Universidad de Pinar del Río "Hermanos Saíz Montes de Oca" (UPR), Cuba; PhD. en Ciencias Económicas por la Universidad de La Habana (UH), Cuba; Máster en Gestión de Empresas Turísticas por la Universidad de Las Palmas de Gran Canarias (ULPGC), España; Experto Universitario en Gestión de Empresas Turísticas por la ULPGC; Docente de la Universidad Regional Autónoma de Los Andes (UNIANDES); Docente Investigador. 